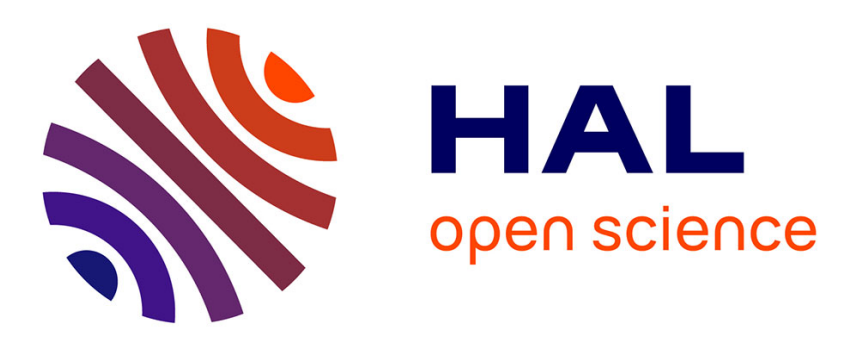

\title{
Sociologie pragmatique et théorie du droit: pour un programme de recherche commun
}

\author{
Lionel Zevounou
}

\section{To cite this version:}

Lionel Zevounou. Sociologie pragmatique et théorie du droit: pour un programme de recherche commun. Droit et Société, 2018, Les violences de genre à l'épreuve du droit, 99, pp.491-504. 10.3917/drs1.099.0491 . hal-01824844

\section{HAL Id: hal-01824844 \\ https://hal.science/hal-01824844}

Submitted on 5 Sep 2018

HAL is a multi-disciplinary open access archive for the deposit and dissemination of scientific research documents, whether they are published or not. The documents may come from teaching and research institutions in France or abroad, or from public or private research centers.
L'archive ouverte pluridisciplinaire HAL, est destinée au dépôt et à la diffusion de documents scientifiques de niveau recherche, publiés ou non, émanant des établissements d'enseignement et de recherche français ou étrangers, des laboratoires publics ou privés. 


\title{
Sociologie pragmatique et théorie du droit : pour un programme de recherche commun
}

\author{
Lionel Zevounou
}

Université Paris Nanterre, UFR de droit et science politique, Bât. F, 200 avenue de la République, F-92001 Nanterre cedex.

<lionel.zevounou@parisnaterre.fr>

\section{À propos de...}

- HeINICH Nathalie, Des valeurs. Une approche sociologique, Paris : Gallimard, NRF, coll. «Bibliothèque des sciences humaines », 2016, 405 p.

Une division du travail arbitraire s'est progressivement imposée entre les juristes et les sociologues. Les premiers sont supposés étudier le droit de «l'intérieur», en recherchant la cohérence des normes d'un système juridique donné. Les seconds proposent un discours sur le droit, compris comme une activité sociale. Cette division du travail est usuellement rendue par la distinction entre un regard " externe modéré » et un regard " externe extrême », hérité des travaux de H.L.A. Hart ${ }^{1}$. Point n'est besoin de revenir en détail sur les débats relatifs au «bon " positionnement épistémique comme si ce dernier pouvait être considéré comme univoque ${ }^{2}$. Grossièrement esquissée, cette division arbitraire du travail a, bon an mal an, tracé une ligne de frontière entre juristes et sociologues, sans toujours parvenir à établir des intérêts épistémiques communs entre les deux communautés. L’ouvrage de Nathalie Heinich dénote dans ce paysage. Alors que l'on était habitué à borner le travail sociologique aux questions jugées « externes » à la discipline (sociologie des jugements ordinaires, sociologie des professionnels du droit, droit et action publique, droit et

1. Le regard " externe modéré » se rapporte à ce que les acteurs considèrent comme norme ; le regard " externe extrême" se concentre, lui, sur le comportement des acteurs. On se contentera de renvoyer, en langue française, au numéro spécial de la Revue interdisciplinaire d'études juridiques issu du colloque qui s'est tenu à Aix, le 26 novembre 2006, consacré à Hart : Hommage à H.L.A. Hart, RIEJ, 57, 2007, p. 1-241 ; pour une analyse plus exhaustive et plus fournie : Gregory BLIGH, Les bases philosophiques du positivisme de H.L.A. Hart, Paris : Fondation Varenne, coll. « collection des thèses », 2017.

2. Pour un aperçu du débat: Thierry DelPeuch, Laurence Dumoulin et Claire De Galembert, Sociologie du droit et de la justice, Paris: Armand Colin, 2014, p. 10-14 ; Reza BANAKAR et Max TrAvers, Law and Social Theory, Londres : Hart Publishing, $2^{\mathrm{e}}$ éd, 2013 ; Liora IsRAËL, "Question(s) de méthodes. Se saisir du droit en sociologue ", Droit et Société, 69-70, 2008, p. 381-395. 
économie) ${ }^{3}$, l'auteur repousse les frontières de l'investigation sociologique au cœur des préoccupations des juristes en abordant de front la question des valeurs. Sa méthode s'inscrit dans l'héritage pragmatiste et analytique ${ }^{4}$; elle constitue une invitation faite aux courants qui se réclament du mouvement Law and Society (réalisme américain, scandinave, droit et économie, socio-legal studies, etc.). Cette méthode se rapproche en particulier de celle que développe, depuis plusieurs années, la tradition analytique du droit. Rapporté au droit, l'emploi de l'adjectif «analytique» requiert quelques nuances avant d'aller plus loin. D'une manière générale, l'usage de l'expression "philosophie analytique » est divers, tant au regard de ses auteurs que des thématiques abordées qui vont de la philosophie de l'esprit à la métaphysique, en passant par la philosophie de la connaissance ou du langage entre autres ${ }^{5}$. Dans le champ juridique en particulier, le terme « analytical jurisprudence » est généralement utilisé par la tradition anglo-américaine. Le sens de l'expression dépasse le clivage français traditionnel «positivisme» / «jusnaturalisme». Le terme théorie analytique du droit auquel nous référons désigne plus modestement le programme de recherche développé depuis plusieurs années à Nanterre au croisement d'une approche anti-cognitiviste et de certains courants d'auteurs italiens, danois et américains qui s'inspirent des travaux de philosophie analytique de la fin XIX $^{\mathrm{e}}$ et début du $\mathrm{Xx}^{\mathrm{e}}$ sur le langage 6 .

Sociologie et théorie analytique du droit, définie dans ce dernier sens, partagent d'ailleurs leur rejet du cognitivisme, auquel il faudrait ajouter, pour la théorie analytique du droit, l'anti-formalisme dans la manière d'appréhender l'activité d'interprétation des normes ${ }^{7}$. En partant de cette observation, la discussion amorcée ici tente de proposer des réflexions d'ordre épistémologique entre l'approche analytique du droit initiée depuis plusieurs années en France et en Italie et le point de vue sociologique proposé par l'auteur. Entendons-nous bien : le livre de Nathalie Heinich ne prend pas pour objet les normes juridiques non plus qu'il discute de la théorie analytique du droit. Il nous a toutefois semblé utile de construire des ponts entre la méthode analytique dont elle se réclame et qui, au fond, pourrait compléter un certain nombre d'interrogations dans le champ de la philosophie du droit positiviste réaliste. Plus largement, les arguments développés par l'auteur apparaissent pertinents

3. Nous reprenons ici le découpage proposé par Thierry DelPeuch, Laurence Dumoulin et Claire DE GALEMBERT, Sociologie du droit et de la justice, op.cit., p. 311-314.

4. Cf. Cyril Lemieux, La sociologie pragmatique, Paris : La Découverte, coll. « Repères ", 2018.

5. Pour un aperçu des orientations de la philosophie analytique en français : Hans-Johann GLOCK, Qu'est-ce que la philosophie analytique?, Paris : Gallimard, coll. «Folio Essais» 2011, (trad. F. Nef) ; Pascal EnGEL (dir.), Précis de philosophie analytique, Paris : PUF, coll. «Thémis Philosophie », 2000.

6. Ainsi, le terme analytical jurisprudence se réfère à des approches théoriques variées, allant de Hume à Hart, Raz, Dworkin ou même des auteurs que l'on qualifierait en France de jusnaturalistes : John Finnis par exemple. Sur ce point, voy. Neil MaCCORMicK, "On Analytical Jurisprudence», in Neil MaCCormick et Ota WeInBerger, An Institutional Theory of Law. New Approaches to Legal Positivism, Dordrecht: Springer, coll. «Law and Philosophy Library », 1986, p. 93-109.

7. Par là il faut entendre l'idée selon laquelle l'activité d'interprétation n'est pas une activité de connaissance, mais de volonté (le juge ne découvre pas le sens de la loi ou les principes qui lui sont sous-jacents, mais décide entre plusieurs significations possibles) : de là découle le programme de recherche qui consiste à comprendre parmi les significations possibles, celle qui a été choisie par l'interprète au regard de ses orientations personnelles, ses valeurs et convictions politiques. 
pour toute approche de sociologie du droit entendue au sens large. Les travaux de Nathalie Heinich trouvent des résonnances à la fois au niveau de l'épistémologie juridique, de la sociologie et de la science du droit. La présente étude tente d'éclairer à cet égard ces différents aspects du champ juridique à la lumière des travaux de l'auteur. Par souci de clarification, on précisera à chaque fois le niveau de langage dont il est question.

L'ouvrage propose une méthodologie sophistiquée d'analyse des valeurs dans les discours ordinaires. L'auteur se livre sur ce point à un travail de clarification bienvenu. Son propos n'entend pas se placer sur un terrain prônant une sacralisation ou un discrédit des valeurs en soi. L'analyse abordée ici ne se réduit pas davantage à l'acception commune de "valeur " au sens d'un prix ou d'une quantification d'un objet, mais plutôt aux biens et principes auxquels est attribuée une valeur. Ainsi, l'ouvrage se concentre à la fois sur les valeurs mobilisées de manière pragmatique pour fonder un jugement sur un objet et sur l'analyse des valeurs sousjacentes à celles mobilisées pour formuler ces différents jugements ordinaires. Ce projet initie les bases d'une grammaire des valeurs, c'est-à-dire une méthode d'analyse qui, pour rendre compte des différents systèmes de valeurs produits par les acteurs sociaux, privilégie l'analyse de leurs discours. Pour le dire dans les mots de Nathalie Heinich : "Étudier la relation que les acteurs entretiennent avec les valeurs, à partir de leurs pratiques de l'évaluation : voilà donc ce qu'il faut entendre par une science sociale des valeurs" (p. 21). S'il est clair que la sociologie de l'auteur diffère sensiblement de celle de Pierre Bourdieu, elle rejoint par certains égards les premières pistes de recherche développées par ce dernier sur le langage ${ }^{8}$.

Si la thématique des valeurs a été abordée par les principaux courants de la sociologie française, leur statut n'a, en soi, pas fait encore l'objet d'un examen rigoureux. L'observation vaut aussi pour le discours savant des juristes. Alors que la norme s'entend d'un ensemble d'énoncés prescriptifs ou d'un impératif d'action, les valeurs "relèvent moins du comportement que des représentations ou, dans d'autres termes, d'une certaine sensibilité » (p. 355) ; les normes sont formulées en « termes déontiques", tandis que les valeurs « sont des concepts axiologiques, qui s'expriment en terme de bien, de mal, de bon ou de mauvais» (p. 355). En d'autres mots, la norme est, en conséquence, tournée vers l'action pendant que la valeur encadre la finalité de cette action. Cette distinction rappelée par Nathalie Heinich n'est pas exclusive de l'idée selon laquelle les valeurs seraient sous-jacentes aux normes. Elle présente simplement l'intérêt d'empêcher toute forme de confusion entre les deux. La finalité de chaque norme fait appel à un ou plusieurs jugements de valeur implicites, en sorte qu'il n'est pas possible de convoquer les valeurs de manière épisodique dans le discours de la science du droit. Aussi convient-il de se prémunir contre une vision consistant à limiter les cas de conflits de valeurs aux seuls "Hard Cases» ou jurisprudences qualifiées de «difficiles». On mettra en garde le lecteur contre une lecture relativement facile en apparence de cet ouvrage, ce qui, dans notre pensée, ne relève pas du reproche, au contraire. Comme beaucoup de travaux

8. Pierre BouRdieu et al., Recherches sur la philosophie et le langage (conférences organisées en 19791980), Groupe de recherches sur la philosophie et le langage, Grenoble, Saint-Martin-d'Hères, 1981, 147 p. 
qui se réclament du courant analytique, la démonstration de Nathalie Heinich apparaît faussement simple. En premier lieu, la thèse proposée éclaire sous un jour différent la manière d'aborder les valeurs dans le discours juridique (I) ; en second lieu, elle suscite des débats primordiaux sur le programme de recherche développé par la tradition analytique du droit (II).

\section{L'étude des valeurs : un objet commun au droit et à la sociologie}

Le droit a ceci de commun avec l'art qu'il fait constamment appel aux jugements de valeur. L'acte d'interprétation en constitue pour le juriste l'une des manifestations par excellence, à travers la définition de la norme comprise comme «signification objective d'un acte de volonté » ${ }^{9}$. La version de 1962 de la Théorie pure $d u$ droit consacre d'ailleurs quelques pages à la distinction normes/valeurs. Hans Kelsen propose à ce titre de distinguer entre jugements de valeur objectifs et subjectifs. Si une norme objectivement valide prescrit un certain modèle de comportement ${ }^{10}$, son effectivité dépend de la conduite qui en résulte; cette conduite peut soit être conforme à la norme (valeur positive), soit s'en éloigner (valeur négative). À partir de là, il est possible de formuler un jugement de valeur dit « objectif » en comparant «l'effectivité » 11 des normes par rapport aux conduites qui en résultent ; on parlera plutôt, afin d'éviter toute confusion, d'une confrontation entre les énoncés normatifs généraux et l'application qui en résulte par les tribunaux. Kelsen ajoute que ces jugements de valeur " objectifs " peuvent être dits vrais ou faux "étant donné qu'ils se rapportent à des normes d'un ordre valable ou en vigueur » : c'est ce qui les distingue des jugements de valeur dits subjectifs où la norme est évaluée par référence à un système de valeur moral extérieur au système juridique examiné 12 .

En France, la question des valeurs a été approfondie par Christophe Grzegorczyk dans son ouvrage La théorie générale des valeurs et le droit publié en $1982^{13}$. Sans revenir sur l'apport de ce travail, son auteur réintègre la question des valeurs en droit dans les débats philosophiques contemporains. Christophe Grzegorczyk tente de trouver un «juste milieu » entre deux extrêmes : une approche "réaliste » - au sens philosophique du terme - qui réifie les valeurs et une approche nominaliste qui, dans sa version radicale, les relègue dans le monde de l'irrationalité, voire les nie. Nathalie Heinich inscrit dans le même sens son analyse des valeurs dans l'évolution des travaux philosophiques contemporains. Ce n'est d'ailleurs pas le moindre des mérites de son travail, tant il semble, d'un point de vue épistémologique, que la philosophie du droit en France, qu'elle se réclame du positivisme ou

9. Hans Kelsen, Théorie générale des normes, Paris : PUF, 1996, coll. «Léviathan », (trad. O. Beaud, F. Malkani), p. 31 ; Sur le pouvoir discrétionnaire du juge : ID., Théorie pure du droit, Paris : LGDJ, Bruxelles : Bruylant, coll. «La pensée juridique », 1999 (trad. C. Eisenmann), p. 335-341.

10. Le terme «modèle de comportement » employé ici s'entend de manière générale et non par référence à la philosophie de Hart.

11. La traduction de 1962 utilise le mot effectivité à la fois dans le sens de l'effectivité du système juridique, mais aussi dans le sens de la conformité d'une norme par rapport aux conduites qui en résultent.

12. Hans KelSEN, Théorie pure du droit, op. cit., p. 27.

13. Christophe GRZEGorCZYK, La théorie générale des valeurs et le droit. Essai sur les prémisses axiologiques de la pensée juridique, Paris : LGDJ, coll. « Bibliothèque de Philosophie du droit », 1982 (préf. M. Villey). 
du jusnaturalisme, a tendance à passer par pertes et profits un certain nombre de justifications nécessaires sur la manière d'appréhender le statut des valeurs dans le discours juridique. La théorie analytique du droit, en particulier, propose de construire un objet généralement assimilé à un discours sur des " faits ». Le discours qui s'élabore au regard de cet objet tente d'analyser, dans leur contexte, les significations retenues par les juges, nous y reviendrons.

Il importe de souligner, encore d'un point de vue épistémologique, que la théorie analytique du droit s'est jusqu'à présent peu empressée d'apporter une justification philosophique au postulat non cognitiviste qui la caractérise dans le champ juridique ${ }^{14}$. Certes, on devine intuitivement qu'un tel choix emprunte à l'héritage de la philosophie émotiviste du début du $\mathrm{Xx}^{\mathrm{e}}$ siècle. Force est pourtant de reconnaître qu'un tel point de vue, aussi légitime qu'il soit, n'est généralement pas justifié dans un contexte où la distinction fait/valeur a pourtant fortement été critiquée ces dernières années dans le champ de la philosophie analytique. Au cœur de ce débat, la question de savoir si l'on peut inférer de la proposition selon laquelle les valeurs constituent un "non-sens" 15 la conclusion d'un subjectivisme radical lorsqu'il s'agit de confronter différentes valeurs entre elles ${ }^{16}$, ou encore le bienfondé d'une certaine interprétation de la « loi » de Hume 17.

Nathalie Heinich s'oppose aux critiques qui prônent la fin de la distinction fait/valeur. Contre une facilité qui consisterait à simplement postuler l'hétérogénéité des mondes descriptifs et évaluatifs, l'auteur défend l'utilité du maintien de la distinction entre faits et valeurs ; cette défense discute les critiques adressées au positivisme logique du début du $\mathrm{xx}^{\mathrm{e}}$ siècle par Hillary Putnam, Richard Rorty, Iris Murdoch ou, en France, Jean-Pierre Cometti. Ces différentes critiques qui tentent de remettre en cause la distinction fait et valeur se heurtent, selon Nathalie Heinich, à

14. Le débat se démarque en ce sens d'une certaine sociologie qualifiée " d'engagée ", mais dont le mérite est d'aborder de front la critique d'une certaine interprétation du concept de neutralité axiologique au nom de la "réflexivité " qui prend au sérieux les interactions entre le chercheur et son objet : Delphine NAUDIER et Maud SIMONET (dir.), Des sociologues sans qualités ? Pratiques de recherche et engagements, Paris : La Découverte, coll. «Recherches», 2011, p. 5-21. Pour une illustration intéressante de ce point de vue : Romain PUDAL, Retour de flammes. Les pompiers, des héros fatigués ? Paris : La Découverte, coll. "L'envers des faits ", 2016, p. 9-10. Pour un point de vue différent: Alain BEITON et Alaïs MARTIN-BAILLON, « La neutralité axiologique dans les sciences sociales. Une exigence incontournable et incomprise", Revue du MAUSS permanente, 18 décembre 2016: <http://www.journaldumauss.net/./?La-neutralite-axiologique-dans-les-1340> (consulté le 16 janvier 2018). Un débat similaire se retrouve dans le champ juridique où la critique d'une certaine forme de neutralité axiologique est remise en cause : Marie-Xavière CATTO, Juliette GATÉ, Charlotte Girard, Stéphanie HennETTE-VAuChez et Carolina Vergel-Tovar, «Questions d'épistémologie : les études de genre sur le terrain juridique ", in Stéphanie Hennette-VAuChEZ, Mathias Möschel et Diane Roman (dir.), Ce que le genre fait au droit, Paris : Dalloz, coll. "À droit ouvert », 2013, p. 3-24 ; dans le même sens : Rafael Encinas de Munagorri, Stéphanie Hennette-Vauchez, Carlos Miguel-Herrera et Olivier Leclerc, L'analyse juridique de $(X)$, Paris : Kimé, 2016.

15. Alfred Jules AYER, Language, Truth and Logic, New York: Dover Publication, 1952 (chap. VI).

16. Une telle justification a été entreprise par Brian LEITER, "Naturalism in Legal Philosophy ", Stanford Encyclopedia of Philosophy, 2012.

17. Voy. sur cette question de l'interprétation de la loi de Hume : Vincent DEsCOMBEs, Le raisonnement de l'ours et autres essais de philosophie pratique, Paris: Seuil, 2007, coll. "La couleur des idées", p. 101-110 ; Alasdair MACINTYRE, Against the Self-Images of the Age. Essay on Ideology and Philosophy, Londres : Dukworth, 1971. 
une objection d'ordre logique. Admettre que les faits et les valeurs se confondent, c'est encore admettre leur homologie. Or, s'il y a homologie entre le monde des faits et celui des valeurs, c'est, comme l'explique Nathalie Heinich ${ }^{18}$, qu'il n'est pas possible de les confondre. Or, comment confondre ou assimiler ce qui est présenté comme identique a priori? Au contraire, admettre l'utilité d'une distinction entre faits et valeurs n'exclut nullement de considérer, qu'en pratique, ils soient tous deux imbriqués dans les discours ordinaires, ceux des juges ou de la doctrine par exemple. Reconnaître cette imbrication ne signifie pas non plus qu'il faille renoncer à produire un discours de connaissance susceptible d'en rendre compte. Il apparaît fondamental de ce point de vue de se doter a minima des outils conceptuels d'une philosophie du langage capable de cerner les multiples formes que prennent les jugements de valeur. Une conséquence en résulte pour la science du droit qui se donne pour tâche de clarifier le discours des juristes.

Considérer à la lumière des débats sur les faits et les valeurs que l'une des spécificités du discours dogmatique produit par les juges ou les universitaires se caractérise par l'assimilation entre propositions et prescriptions apparaît comme une condition nécessaire, mais insuffisante. Nécessaire, car il convient de rendre compte de la spécificité d'une science du droit qui ne se donne pas toujours pour modèle une forme d'objectivité scientifique. L'affirmation de cette spécificité propre à la dogmatique ne permet pas pour autant d'en pénétrer la «boîte noire " : de ce point de vue, s'en tenir à l'idée selon laquelle la dogmatique ne distingue pas entre proposition et prescription apparaît insuffisant au regard de l'analyse du langage. Et ce, quand bien même la théorie analytique du droit s'est aussi attachée à déconstruire les raisonnements des juges.

Il faudrait sans doute y adjoindre un certain nombre d'apports de la philosophie du langage. C'est ce que tente de faire Nathalie Heinich dans le domaine de l'art et que l'on pourrait aisément transposer dans l'analyse du discours juridique en mobilisant les apports de John Searle ou Richard Mervyn Hare ${ }^{19}$. Dans la famille des jugements de valeur, ce dernier distingue le jugement de goût « explicitement subjectif» du jugement de valeur "à visée objectivante»20. Entre ces deux types d'énonciations, des raffinements sont possibles : selon les contextes, le jugement de goût exprimé par un profane n'a pas la même force de persuasion qu'un jugement d'expert. Une telle différence naît précisément de la compétence de l'énonciateur. La réflexion qu'engage Nathalie Heinich suscite en ce sens l'intérêt : le discours de la dogmatique doit-il être mis sur le même plan que l'opinion ordinaire 21 ? Sinon, comment parvenir à identifier sur quoi se fonde son autorité, quels en sont les ressorts? Ici encore, la mobilisation des travaux de John Searle et d'autres - Oswald Ducrot ou Gilbert Dispaux - ouvre une piste de réflexion intéressante. La gradation

18. Nathalie HeInICH, Des valeurs. Une approche sociologique, op.cit., p. 111.

19. R. M. HARE, The Language of Morals, Oxford : Clarendon Press, 1952.

20. Nathalie HeINICH, Des valeurs. Une approche sociologique, op.cit., p. 116.

21. Pour une présentation de quelques débats sur le raisonnement juridique dans la philosophie du droit américaine: Pierre BRunet, "Le raisonnement juridique dans tous ses états ", Droit et Société, 83, 2013, p. 193-202. 
nuancée du fait au jugement de valeur que propose Searle apparaît utile à plus d'un titre. Deux catégories de faits, les faits dits «bruts » et les faits dits "épistémiques" ou faits sociaux sont croisés avec deux catégories de jugements : les jugements objectifs («le mont Everest se situe dans l'Himalaya») et les jugements subjectifs («le mont Everest est magnifique»). Searle propose de combiner les catégories de faits (bruts et sociaux) avec les catégories de jugement (objectif et subjectif). Ainsi, affirmer, à propos des jugements subjectifs, que : "le mont Everest est plus beau que le mont Whitney" est un jugement épistémiquement subjectif sur une entité ontologiquement objective, tandis que "j'éprouve en ce moment une douleur au bas du dos" est un jugement épistémiquement objectif sur un fait ontologiquement subjectif » 22 . La catégorie de l'énoncé épistémiquement objectif sur un fait ontologiquement subjectif est caractéristique du jugement de valeur à visée objectivante. C'est le croisement des faits et des jugements qui débouche sur une gradation d'énoncés, dont le jugement de valeur. Au sein des jugements de valeur, l'analyse ouvre dès lors la voie à une complexité qui dépasse la catégorie usuelle de "prescriptions » utilisée par le positivisme analytique.

L'enjeu apparaît d'importance pour la science du droit, afin de ne pas réduire la dogmatique ou la justification des acteurs du système juridique à de simples «jugements de valeur ", compris comme l'expression de choix politiques voire idéologiques, dissimulés derrière l'évaluation ou l'interprétation des normes. De là, il serait facile d'en tirer la conclusion hâtive selon laquelle le discours des acteurs juridiques ne serait en définitive que le paravent d'une forme d'exercice du pouvoir, ce qui présenterait le risque de délaisser l'analyse de la manière dont se construit "l'objectivation " d'une telle forme discursive. Les deux sont pourtant liées. Un des ressorts de l'autorité du discours juridique tient précisément dans son formalisme et son contenu linguistique. Qu'ils soient politiques ou tentent de dissimuler des idéologies, la formulation des prescriptions doctrinales par les acteurs juridiques tend vers une objectivité linguistique apparente, ce que démontre Nathalie Heinich lorsqu'elle aborde la question de l'expertise dans le domaine de l'art ${ }^{23}$. Le discours de l'expert est le siège d'une tension entre le subjectif et l'objectif ${ }^{24}$. Cela implique d'admettre que, dans certaines circonstances, l'expert sait faire la part entre le jugement de valeur et le jugement de fait. Ainsi, «les acteurs, même s’ils ont - plus ou moins nettement - conscience de la spécificité du jugement de valeur [...] par rapport au jugement de fait, n'ont pas forcément intérêt à la respecter. C'est là [pour reprendre Bourdieu] le propre du "sens pratique", que méconnaissent les théoriciens trop attachés aux "choses de la logique" au détriment de la "logique des choses" » (p. 127).

22. Nathalie HeINICH, Des valeurs. Une approche sociologique, op.cit., p. 122-123.

23. Ce que démontre aussi Bourdieu, non pas en se fondant sur les apports de la philosophie du langage, mais bien par l'identification de la singularité professionnelle et du capital symbolique des juristes : Pierre BouRdieu, " La force du droit. Éléments pour une sociologie du champ juridique ", Actes de la recherche en sciences sociales, 64,1986, p. 3-19.

24. Nathalie HEINICH, Des valeurs. Une approche sociologique, op.cit., p.77-104. 
L'objectivation du discours de l'expert s'appuie sur diverses ressources (recherche de cohérence par rapport aux décisions antérieures, respect des procédures conventionnellement admises, recours à la comparaison ou à la collégialité, connaissance de l'histoire de l'objet analysé, etc.) Aussi, l'évaluation de l'expert - qu'il s'agisse du juge, de la doctrine ou de l'expert en art - ne peut-elle se réduire à un simple jugement de valeur du type « jugement de goût arbitraire, contingent et non justifiable ${ }^{25}$. Si, sur la question de l'analyse de la forme du jugement de l'expert, les arguments développés par Nathalie Heinich ne sont pas véritablement nouveaux, en revanche, la gradation des jugements de valeur qu'elle propose paraît entamer une réflexion novatrice dans le champ de la théorie analytique du droit lorsqu'il s'agit de saisir plus finement à la fois la structure argumentative et l'autorité des discours dogmatiques selon les contextes.

\section{Quel point(s) de vue(s) adopter pour l'analyse des valeurs : déconstruire et/ou comprendre?}

Comme il a été évoqué précédemment, la théorie analytique du droit, développée en particulier à Nanterre, privilégie un point de vue non cognitiviste ; elle partage cette approche avec l'ensemble des courants positivistes dans le champ juridique. La séparation linguistique entre les normes et le discours-objet sur les normes, encore appelé science du droit, apparaît primordiale pour décrire l'objet étudié par la théorie du droit analytique ${ }^{26}$. Nathalie Heinich ne dit pas autre chose lorsqu'elle revient sur l'utilité de séparer engagement et distanciation : "Ce n'est pas en s'engageant dans les controverses que le sociologue peut en éclairer les tenants et les aboutissants, mais, au contraire, en se détachant de tout jugement de valeur des acteurs qu'il étudie. C'est dire que, plus que pour tout autre objet, le projet d'une sociologie des valeurs exige, pour être mené à bien, une condition fondamentale, logée dans la posture adoptée par le chercheur: l'abstention de toute prise de position à l'égard tant des objets évalués par les acteurs que des jugements de valeur proférés par eux 27 ». Nul doute que, d'un point de vue méthodologique, Hans Kelsen rejoint Max Weber quant à la poursuite d'un idéal d'objectivité qu'il faudrait ne pas confondre avec tout refus d'engagement politique ${ }^{28}$. La recherche

\section{Ibid., p. 81.}

26. Michel Troper, Pour une théorie juridique de l'État, Paris : PUF, coll. « Léviathan », 1994, p. 36.

27. Nathalie HeINICH, Des valeurs. Une approche sociologique, op.cit., p. 17

28. On évitera la tentation de faire usage de concept wébérien de neutralité axiologique dans la mesure où, comme les travaux d'Isabelle Kalinowski l'ont montré, la traduction introduite par Julien Freund de « neutralité axiologique " comme synonyme à la fois d'objectivité et de modération dans l'engagement intellectuel a largement été influencée par Raymond Aron. Or lorsque Weber parle de " neutralité axiologique ", ce n'est pas pour s'opposer à l'existence ou à l'adhésion de valeurs, mais bien pour dénoncer « l'usage malhonnête qui peut être fait des valeurs lorsqu'elles sont présentes sans être données, comme telles, lorsqu'elles sont masquées, cachées [...]. La Wertfreiheit, dont le sens exact serait la non-imposition des valeurs, doit selon lui prévaloir dans le domaine des sciences et de la connaissance, par opposition aux domaines de la croyance (religieuse, politique, etc.) régis par la propagande, et dans lesquels on cherche à imposer des valeurs par tous les moyens. La situation de transmission que Weber a en tête est très concrète : c'est la situation d'enseignement, classiquement décrite dans la conférence sur la science ", in Isabelle KALINOWSKI 
d'objectivité assimilée à tort à une posture de « neutralité axiologique » souvent mal comprise a fait craindre en droit comme en sociologie l'émergence d'une sorte de caution passive face aux horreurs de l'histoire. Sur ce point, la critique de Léo Strauss évoquée par Nathalie Heinich rejoint celle qu'a pu adresser en son temps Danièle Lochak au positivisme juridique sous Vichy.

Là-dessus, sociologie et une certaine philosophie analytique se rejoignent: la posture du chercheur ne doit pas être confondue avec l'opinion du citoyen ordinaire, aussi engagé et éclairé soit-il ${ }^{29}$. Rendre compte des ressorts d'un système juridique totalitaire ne signifie pas nécessairement que l'on adhère aux valeurs qui en sont issues. On touche ici à des différences sensibles entre traditions académiques. Alors qu'en France la figure de l'intellectuel "engagé » est généralement associée au "progressisme », il n'en est pas de même dans d'autres pays, notamment l'Allemagne. En attestent les écrits «engagés» de Carl Schmitt qui démontrent, s'il fallait le rappeler, l'idée illustrée par Nathalie Heinich, selon laquelle: «La science allemande se posait en antidote du faux idéal de l'objectivité et la plus belle formulation d'une science idéologique nous vient d'un personnage aussi important que feu le Dr Goebbels en personne : la vérité est ce qui sert le peuple allemand ${ }^{30}$. " La référence à un engagement ne peut, dans le champ académique - qui bénéficie d'une présomption apparente de neutralité -, faire l'économie d'une clarification des registres de discours mobilisés (scientifiques/engagés). La posture critique ne peut à elle seule tenir lieu de science, en droit comme en sociologie.

Nathalie Heinich met en garde contre le risque d'une telle confusion dans le domaine de la sociologie, mais cette remarque pourrait aussi valoir pour la production du discours dogmatique des juristes. Les frontières poreuses entre la posture du juriste savant et celle du juriste « engagé » 31 ne sont pas toujours favorables aux clarifications d'ordre méthodologique. Aussi, la nécessité de proposer un critère intellectuel de démarcation entre la « science» et le discours d'expert constitue-t-elle une exigence épistémique permettant de faire la part entre science, propagande ou engagement politique. S'interroger sur la méthode adoptée et les présupposés qu'elle implique participe aussi à rendre le champ des sciences sociales moins poreux à l'emprise du pouvoir politique. Dans la pratique dogmatique ordinaire, le discours des juristes savants en particulier reste généralement rétif à toute forme de tentative explicite de clarification relative à ce que produire un discours de «connaissance»

et Max WEBER, La science, profession \& vocation. Suivi de leçon wébériennes sur la science et la propagande, Paris : Agone, 2006, coll. « Banc d'essais », p. 199 (voy. en particulier le chapitre IV).

29. On renvoie sur ce point aux références mentionnées à la note de bas de page $n^{\circ} 8$. À quoi il faut ajouter plusieurs controverses récentes dans le champ de la sociologie sur cette question de la neutralité de l'universitaire ; l'un des derniers numéros de la revue Le Débat (197, 2017, p. 113-141) a accueilli plusieurs auteurs (Olivier Galland, Gérald Bronner, Étienne Gehin, Pierre-Michel Menger), dont Nathalie Heinich, affirmant leur attachement à une forme de neutralité axiologique, entendue au sens de critères permettant de contrôler la production de la science et d'appréhender la complexité du social. Une réponse de Luc Boltanski et Arnaud Esquerre a été publiée dans Le Monde du 23 novembre 2017.

30. Nathalie HEINICH, Des valeurs. Une approche sociologique, op.cit., p. 115 voy. aussi : Carl SCHMITT, « La science allemande du droit dans sa lutte contre l'esprit juif. 1936 », Cités, 14, 2003, p. 173-180.

31. On entend par là à la fois le juriste engagé pour une cause, quelle qu'elle soit, ou le juriste engagé dans le champ du pouvoir politique ou économique en qualité de conseiller du prince ou de praticien. 
sur le droit veut dire ${ }^{32}$. Si l'on délaisse les approches convenues sur les grands courants de la pensée juridique (jusnaturalisme, positivisme, etc.) pour se concentrer sur la science en train de se faire, le faible questionnement relatif aux méthodes d'appréhension du droit interroge les valeurs sous-jacentes à l'évaluation scientifique produite par les pairs. En prenant au sérieux le tournant pragmatique qui s'est opéré en philosophie des sciences et en sciences sociales ${ }^{33}$, les travaux, cités par Nathalie Heinich, de Michèle Lamont dans le champ de l'économie pourraient contribuer à alimenter la réflexion permettant d'élucider un tel manque de prise en considération de questions d'ordre méthodologique. La science du droit gagnerait, ce faisant, à étendre son programme de recherche focalisé sur l'analyse des discours juridiques pour se concentrer sur les ressorts de la production scientifique ordinaire. La distinction entre "valeurs publiques » et "valeurs privées » reprise par Nathalie Heinich aux travaux de Michèle Lamont pourrait servir de base à l'analyse des pratiques émanant d'enceintes chargées de l'évaluation scientifique dans la production d'un discours savant sur le droit. Sur quoi repose l'évaluation des pairs? Est-il possible, en se focalisant sur de telles évaluations, de mieux cerner l'éthos scientifique des juristes universitaires ? L'utilité de la distinction entre "valeurs publiques » et «valeurs privées » se justifie en ce sens. Les premières se rapportent en substance à une reconstruction ex post du jugement scientifique, celle qui figure généralement dans les rapports d'évaluation adressés, selon les publics, à des candidats ou des collègues; les secondes réfèrent aux critères extra scientifiques : ceux qui ne sont pas considérés comme "admissibles" au regard d'une certaine éthique de la science. Ainsi, alors que les "valeurs publiques " se rapportent aux critères formels de l'objet scientifique examiné, les «valeurs privées » se focalisent davantage sur les "pratiques effectives » (p. 88-89), celles qu'il n'est pas possible de faire figurer dans un jugement d'ordre scientifique, mais qui participent quand même de l'évaluation proposée. Nathalie Heinich ouvre, ce faisant, des perspectives fort utiles pour la science du droit à l'analyse critique du discours savant des juristes, qui permettent de cerner avec plus de précision le sens du concept de «science normale » ou normalisée dans le champ juridique. Examiner ce que signifie la science ordinaire du droit impliquerait dès lors de procéder à une sociohistoire de la manière dont les juristes se représentent leur propre savoir.

Si la sociologie analytique proposée par Nathalie Heinich partage avec le positivisme épistémologique le même souci d'objectivité, plusieurs nuances s'imposent. La théorie analytique du droit n'a pas tranché encore explicitement entre déconstruire et

32. Voy. en ce sens : Éric MiLlard, "De l'aveu théorique comme préalable au travail juridique savant ", Actes du $\mathrm{VI}^{\mathrm{e}}$ congrès de l'Association française de droit constitutionnel, Université de Montpellier, 2005 : $<$ https:// halshs.archives-ouvertes.fr/halshs-00009961> (consulté le 20 décembre 2017).

33. On renvoie en ce sens à la livraison de la revue Raisons pratiques: Francis CHATEAURAYNAUd et Yves COHEN (dir.), « Histoires pragmatiques », 25, 2016, Paris : éditions EHESS. On renvoie aussi aux travaux qui se développent depuis plusieurs années, dans le domaine de la sociologie, et qui abordent la science comme une activité sociale à part entière et non un savoir mythifié : Cetina Karin KNorR, Epistemic Cultures. How Sciences Make Knowledge, Cambridge : Harvard University Press, 2003 ; Thomas GIERYN, Cultural Boundaries of Science: Credibility On the Line, Chicago : University of Chicago Press, 1999. 
comprendre $^{34}$. Déconstruire suppose un regard différent de l'ordinaire : un regard qui entend mettre en lumière ce qui se passe en "coulisse". Différemment, comprendre nécessite de se mettre à la place des acteurs en vue de reconstruire le sens de leurs actions. Autrement dit, l'appareil critique développé depuis plusieurs années par la théorie analytique du droit vise-t-il à dénoncer ou à comprendre la production des normes et des discours sur les normes? Un auteur a relevé, à juste titre selon nous, l'ambiguïté entre une approche « sociologisante" et la construction d'un objet d'étude empirique qui ne serait qu'en partie sociologique - la jurisprudence, entendue ici comme l'étude critique du raisonnement juridique et de ses justifications ${ }^{35}$. Que faut-il tirer de cette critique quant à la construction d'un objet empirique ? On se contentera de formuler quelques questions à cet égard. Du point de vue de sa définition, qu'implique l'analyse des actes de signification dans leur contexte immédiat ? La description du processus de décision judiciaire doit-elle être appréhendée d'un point de vue compréhensif ou simplement linguistique? Peut-on, dans les deux cas, se réclamer d'une démarche sociologique ou empirique sans au préalable enrichir le travail de déconstruction, auquel procède habituellement la théorie analytique du droit, d'outils conceptuels et d'une méthodologie susceptibles de parvenir à mieux cerner les différents faits sociaux liés à l'interprétation des normes ou plus largement à la production des énoncés juridiques? En somme, une des réflexions majeures qu'engage l'ouvrage de Nathalie Heinich, à la suite de certains travaux en sociologie du droit, interroge l'opportunité d'ouvrir plus largement les outils conceptuels de la théorie analytique du droit aux sciences sociales d'un point de vue méthodologique.

Il faut maintenant discuter pour finir un autre aspect de l'ouvrage de Nathalie Heinich qui distingue entre valeurs et intérêts : «Les valeurs sont-elles des entités autonomes, des motivations authentiques, ou bien ne sont-elles que la traduction voire la dissimulation d'intérêts" (p. 349) ? La théorie analytique du droit dans sa version développée en France n'a, il faut le reconnaître, pas davantage clarifié cette question. Une des propositions fortes que cette théorie développe est que l'acte d'interprétation constitue un acte de volonté, déterminé parmi de multiples significations possibles ${ }^{36}$. La conséquence de cette proposition a déjà été évoquée ailleurs : elle permet de se focaliser sur la production des significations et non plus les significations elles-mêmes ${ }^{37}$. Un tel déplacement aboutit à considérer le système juridique comme une forme particulière d'exercice du pouvoir : « la description de la structure de l'ordre juridique est en même temps une description de la structure du pouvoir politique [...] ; une analyse de la distribution du pouvoir ${ }^{38}$. Cette proposition permet de mieux saisir l'idée selon laquelle, si les normes constituent la signification objective d'actes de volonté, il est possible de déconstruire les présupposés

34. Sur la délicate distinction entre expliquer et comprendre: Caherine CoLLIOT-THÉLÈNE, «Expliquer/ comprendre : relecture d'une controverse», Espaces Temps, 84-86, 2004, p. 6-23.

35. On renvoie sur ces questions au travail pionnier d'Évelyne SERVERIN, De la jurisprudence en droit privé : théorie d'une pratique, Lyon : Presses Universitaires de Lyon, coll. « Critique du droit », 1985.

36. Michel Troper, Pour une théorie juridique de l'État, op.cit., p. 87.

37. Pierre Brunet, « Michel Troper et la “théorie” générale de l’État. État général d'une théorie », Droits, 37, 2003, p. 87-110, spéc. p. 96.

38. Michel Troper, Pour une théorie juridique de l'État, op.cit., p. 67. 
sous-jacents à leur interprétation. Fort de ce nouveau programme de recherche, une ébauche d'analyse des contraintes juridiques a été développée ${ }^{39}$. Si l'on admet que les acteurs juridiques cherchent à masquer leur pouvoir derrière les justifications apportées aux normes, faut-il en conclure que le concept de «droit » développé par la théorie analytique depuis plusieurs années ne vise qu'à rendre compte des stratégies diverses développées par les interprètes pour asseoir leur pouvoir? Qu'en est-il de la possibilité de rendre compte des valeurs sous-jacentes à ces normes?

Une critique majeure a été adressée en ce sens à la théorie analytique, celle d'un scepticisme - voire d'un nihilisme exacerbé - à l'égard de la possibilité même de "connaître" la signification des normes 40 . De toute évidence, le constructivisme porté par la théorie analytique du droit rejoint un mouvement fructueux opéré dans les sciences sociales depuis plusieurs années : celui d'affirmer le caractère non naturel d'un certain nombre de réalités sociales (nation, race, genre, droit, etc.). Il est difficile de contester l'apport de cette approche dans le champ des sciences sociales, y compris juridiques. Plus discutable en revanche est l'ambition scientifique que se donne la théorie analytique du droit. Pour le dire à partir des catégories de Bernard Williams ${ }^{41}$, s'agit-il de récuser toute idée de vérité (il n'est pas possible de connaître le contenu des normes) ou simplement de discuter la véracité d'un certain nombre de représentations dans les discours normatifs et celui des juristes (comprendre la naturalisation des catégories de "contrat», "propriété ", par exemple) ? En réduisant le «droit » à des intérêts, idéologies ou stratégies, la théorie analytique du droit ne risque-t-elle pas de sombrer, à l'instar des théories marxistes, utilitaristes ou bourdieusienne avant elles, dans une forme de déterminisme inavoué ? Le programme critique proposé par la théorie analytique du droit se donne-t-il pour objectif de critiquer en vue de dénoncer dans un sens politiquement engagé ou critiquer en vue de rendre compte et d'expliquer?

C'est ici que le concept de compréhension sociologique s'avère d'une utilité certaine. Il est en effet possible d'échapper à l'accusation de scepticisme radical à l'égard du discours normatif, en tenant compte des critiques adressées depuis plusieurs années aux théories qualifiées de déterministes qui ont tendance à ne voir dans l'invocation des valeurs qu'une dissimulation d'intérêts variés. La question se pose alors: comment prendre en compte l'étude des valeurs dans les discours normatifs ou dogmatiques tout en évitant à la fois le piège du déterminisme, la confusion des valeurs et de morale ou le réductionnisme individualiste? En s'appuyant sur les travaux de Randall Collins, Nathalie Heinich propose, pour surmonter cette difficulté, une acception plus large du concept « d'intérêt ». Il est tout à fait possible dans ce dernier cas d'admettre que les acteurs juridiques agissent en fonction de valeurs communes, de "positionnement hiérarchique » ou simplement par hédonisme. L'admettre ne signifie nullement prendre part aux jugements de

39. Véronique ChampeIL-Desplats, Christophe GrZegorczyK et Michel Troper, Théorie des contraintes juridiques, Paris : Bruylant/LGDJ, coll. «La pensée juridique », 2005.

40. Otto Pfersmann, "Contre le néo-réalisme juridique. Pour un débat sur l'interprétation », Revue française de droit constitutionnel, 50 (2), 2002, p. 279-334.

41. Bernard WiLliams, Vérité et véracité : essai de généalogie, Paris : Gallimard, coll. « NRF Essais », 2006. 
valeur dont on rend compte, mais introduit plutôt une causalité explicative plurielle - tenant compte à la fois des intérêts et des valeurs - du comportement des acteurs juridiques. La théorie analytique du droit gagnerait à s'inscrire pleinement dans un programme de recherche sociologique qui prenne au sérieux les valeurs, mais en propose aussi une "grammaire» plus précise. Trois avantages en découlent. Le premier permettrait d'enrichir le programme de la théorie analytique du droit dans une philosophie du langage capable d'identifier les valeurs qui guident l'action des juges et du législateur sans les confondre avec les normes qui en sont le produit. Un second avantage consisterait à compléter la tâche de la science du droit entendu comme l'étude du raisonnement juridique - par un travail sociologique à part entière sur l'identification des différents registres de valeurs dans le langage des juristes informés par d'autres données empiriques (statistiques judiciaires, enquêtes qualitatives). Mieux encore, il convient de chercher à comprendre de quelle manière se forment les jugements de valeur comme l'invite à faire Nathalie Heinich. Se limiter à un travail de clarification compris comme une mise à nu des présupposés du juge ou de la doctrine ne peut suffire. Le travail de "dévoilement» doit s'accompagner d'une tentative de reconstitution du sens subjectif de l'activité des interprètes 42 . Ce second volet intègre de plain-pied le champ de la sociologie juridique dominante : il permettrait de mieux cerner des questions utiles à la connaissance juridique et qui échappent parfois au travail du sociologue.

Comprendre dans un premier temps l'objet du travail des juristes savants importe pour la science du droit, de ce point de vue. Cette tâche est plus complexe qu'elle n'en a l'air. Les juristes travaillent certes sur des données textuelles (lois, jurisprudences, règlements, constitution), mais de quelle manière est-il possible de systématiser plus finement leur pratique ? Si l'on prend l'exemple de l'objet « jurisprudence », faut-il comprendre la jurisprudence étudiée et commentée par la doctrine, c'est-à-dire celle qui en amont est préalablement sélectionnée par l'administration judiciaire sous le contrôle des cours suprêmes? D'où proviennent ces valeurs communes qui consistent à mettre en cohérence des jurisprudences rendues dans des contextes différents, sans jamais prendre la peine d'ordonner l'analyse des cas en fonction des années, des chambres et des formations de juridiction? La contrainte selon laquelle les juges du fond prennent des décisions par crainte de l'interprète authentique mérite elle aussi une analyse contextuelle prenant au sérieux les cultures propres à l'ordre administratif et judiciaire (le lien entre le management de la justice et la production des décisions de justice, l'identification des stratégies argumentatives menées par les juges du fond pour éviter la censure de l'interprète authentique, etc.).

Si ces différentes questions ne sont pas abordées par Nathalie Heinich, elles méritent en tout cas une réflexion d'ampleur. Les réduire à des débats qui opposent analyse externe et/ou interne n'apparaît pas pertinent d'un point de vue épistémique ; disqualifier cette approche comme n'étant pas du droit ne l'est pas davantage pour ceux qui partagent et assument la conviction selon laquelle le droit et les juristes ont toute leur place dans le champ des sciences sociales.

42. Cette approche a aussi été développée par Luc BOLTANSKI et Laurent THÉVENOT, De la justification: les économies de la grandeur, Paris : Gallimard, coll. « NRF Essais », 1991. 
- L'auteur

Lionel Zevounou, maître de conférences en droit public à l'Université Paris Nanterre, est chercheur au Centre de Théorie et Analyse du droit (UMR 7074) et chercheur associé à l'IDHES (UMR 8533). Ses recherches portent sur les rapports droit-économie d'un point de vue sociologique et critique. Il a notamment publié :

— "L'Afrique dans le monde" et "le monde en Afrique" : jalons pour un programme de recherche pluridisciplinaire sur le droit ", Bulletin du CODESRIA (à paraître) ;

— «Le concept de constitution économique. Une analyse critique», Jus Politicum. Revue de droit politique, 20, 2018 ;

- «(Re) penser le droit administratif avec Jacques Caillosse », Revue de droit. Université de Sherbrooke, 46 (1), 2016. 


\section{Dossier}

Les violences de genre à l'épreuve du droit

coordonné par Marta Roca i Escoda, Pauline Delage, Natacha Chetcuti-Osorovitz

Marta Roca i Escoda, Pauline Delage,

Natacha Chetcuti-Osorovitz

Delphine Lacombe

Solenne Jouanneau, Anna Matteoli

Isabelle Carles

Océane Pérona

Geraldine Brown
Quand la critique féministe renouvelle le droit. Présentation du dossier

Légiférer sur les «violences de genre» tout en préservant l'ordre patriarcal. L'exemple du Nicaragua (1990-2017)

Les violences au sein du couple au prisme de la justice familiale. Invention et mise en cuvre de l'ordonnance de protection

Les politiques de lutte contre les violences de genre en Belgique et les femmes migrantes : entre volonté de protection et contrôle migratoire

Déqualifier les viols : une enquête sur les mains courantes de la police judiciaire

De la «violence masculine» à la «gestion des menaces». Judiciarisation des violences et euphémisation des rapports sociaux de sexe

\section{Études}

Prune de Montvalon

Habibou Fofana
Sous condition « d'émancipation active» : le droit d'asile des prostituées nigérianes victimes de traite des êtres humains

Rapprocher la justice des justiciables. Une ethnographie de la «distance judiciaire» au Burkina Faso

\section{Traduit pour vous}

Susanne Baer

Le droit comme pratique. Les défis de la recherche juridique aujourd'hui

Bernard S. Jackson

Le périple sémiotique d'un théoricien du droit

\section{À propos}

Marc Joly

Lionel Zevounou

Guillaume Richard
La révolution sociologique : réponse à Federico Tarragoni Sociologie pragmatique et théorie du droit : pour un programme de recherche commun

Défendre la forme du droit. Regards contemporains sur le droit nazi

\section{Chronique bibliographique}

$$
\text { Prix : } 60 €
$$

ISBN 978-2-275-02912-2

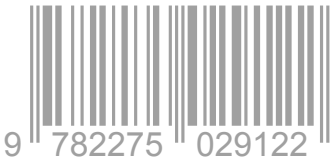

RESEAU EUROPEEN DE COMMUNICATION ENTRE REVUES D'ETUDES SUR LE JURIDIQUE HERMES 